\title{
Desenvolvimento e Validação de uma Escala de Estratégias de Aprendizagem no Trabalho
}

\author{
Learning Strategies at Work: Development and Validation of a Scale
}

\author{
Hugo Pena Brandão*,a,b \& Jairo Eduardo Borges-Andrade \\ ${ }^{a}$ Fundação Getúlio Vargas, Brasília, Brasil, ${ }^{b}$ Banco do Brasil, Brasília, Brasil \\ \& ${ }^{c}$ Universidade de Brasília, Brasília, Brasil
}

\begin{abstract}
Resumo
Este estudo objetivou desenvolver uma escala de estratégias de aprendizagem no trabalho e verificar a freqüência com que estas são utilizadas por gestores de um banco público. Os dados foram coletados por meio de levantamento documental, entrevistas e questionários. Estes foram respondidos por 881 gestores da empresa e os dados foram submetidos a análises descritiva e fatorial. Os resultados revelaram 26 práticas utilizadas para aprender no trabalho, agrupadas em cinco fatores: reflexão ativa; busca de ajuda interpessoal; busca de ajuda em material escrito; reprodução; e aplicação prática $(\alpha=0,79$ a 0,92$)$. As estratégias de aprendizagem mais utilizadas pelos respondentes foram a busca de ajuda interpessoal e a reflexão. Ao final, são apontadas direções para realização de novas pesquisas.

Palavras-chave: Estratégias de Aprendizagem no Trabalho; Escala de Medida; Análise Fatorial.

Abstract

This study had the purpose of developing a scale of learning strategies at work and verifying how often they are used by managers of a Brazilian public bank. Data collection was made through documental search, interviews and structured questionnaires. The questionnaires were answered by 881 bank managers and the data were submitted to descriptive and factor analysis. Results revealed 26 practices to learn at work which were grouped into five factors: active reflection; interpersonal help seeking; seeking help from written material; reproduction; and practical application $(\alpha=.79$ to .92$)$. The most used learning strategies were interpersonal help seeking and active reflection. At last, an agenda for future research is suggested.

Keywords: Learning Strategies at Work; Scale of Measurement; Factor Analysis.
\end{abstract}

O desenvolvimento de competências profissionais ocorre por meio da aprendizagem, seja ela decorrente de ações formalmente estruturadas pelas organizações ou de práticas informais adotadas pelos próprios empregados para promover a aquisição de conhecimentos, habilidades e atitudes no trabalho (Brandão \& BorgesAndrade, 2007).

A complexidade e a dinâmica do ambiente organizacional geram diversificadas necessidades de competências, fazendo com que o provimento aos empregados de freqüentes oportunidades de aprendizagem constitua um desafio para as organizações (Casey, 1999).

Em decorrência, tem sido cada vez mais comuns, tanto no meio acadêmico quanto no ambiente organizacional, a realização de pesquisas para identificar e analisar os processos pelos quais as pessoas aprendem e desenvolvem

"Endereço para correspondência: Universidade Corporativa Banco do Brasil, Diretoria Gestão de Pessoas, Setor de Clubes Esportivos Sul, Trecho 2, Lote 22, Ed. Presidente Tancredo Neves, $1^{\circ}$ andar, Brasília, DF, Brasil, CEP 70200-002.E-mails: hugopb@tba.com.bre jairo@unb.br suas competências (Brandão, 2008). Entre tais pesquisas, há aquelas dedicadas ao estudo de estratégias de aprendizagem no trabalho, ou seja, de práticas informais que os indivíduos utilizam para auxiliar a aquisição de conhecimentos e habilidades em seu próprio local de trabalho (Holman, Epitropaki, \& Fernie, 2001; Pantoja \& BorgesAndrade, 2009; Sonnentag, Niessen, \& Ohly, 2004).

O presente estudo objetivou desenvolver e validar uma escala de medida de estratégias de aprendizagem no trabalho, bem como verificar a freqüência com que tais estratégias são utilizadas por gestores de um banco público brasileiro. O desenvolvimento dessa escala é particularmente importante para que futuras pesquisas possam verificar se o uso dessas estratégias de aprendizagem está associado ao desenvolvimento de competências, ao desempenho no trabalho e a outras medidas do comportamento organizacional.

\section{Aprendizagem nas Organizações}

Aprender implica em modificar ou desenvolver conhecimentos, habilidades e atitudes. Constitui uma mudança relativamente duradoura na capacidade ou no comporta- 
mento da pessoa, transferível para novas situações com as quais ela se depara (Gagné \& Medsker, 1996). A aprendizagem, então, pode ser vista como um processo dinâmico, que gera mudanças na forma pela qual uma pessoa vê, experimenta, entende e conceitua algo (Matthews \& Candy, 1999). No contexto organizacional, o processo de aprendizagem tem como finalidade promover mudanças nos domínios cognitivo, psicomotor e atitudinal (Sonnentag et al., 2004), podendo ocorrer tanto para responder a necessidades correntes de trabalho como para desenvolver competências relevantes para o futuro (Abbad \& Borges-Andrade, 2004).

A aprendizagem pode ser classificada como: implícita (também chamada natural), quando não existe o propósito deliberado de aprender e nem a consciência de que se aprende; ou explícita, quando for decorrente de uma atividade deliberada e consciente (Pozo, 2002). Nas organizações, as atividades de aprendizagem explícita, por sua vez, podem ser (Sonnentag et al., 2004): formais, quando forem estruturadas e constituírem iniciativa empreendida ou apoiada pela organização, como é o caso da realização de cursos, seminários e orientação formal; ou informais, quando não forem estruturadas e constituírem iniciativa do próprio empregado, com ou sem o apoio da organização, como ocorre no intercâmbio informal de conhecimentos e na aprendizagem por tentativa e erro (experimentação).

Como a aprendizagem implica mudar ou desenvolver conhecimentos, habilidades e atitudes pode, por conseguinte, promover o desenvolvimento de competências profissionais. Tais competências, segundo Freitas e Brandão (2006), podem ser entendidas como combinações sinérgicas de conhecimentos, habilidades e atitudes, expressas pelo desempenho do indivíduo dentro de determinado contexto organizacional, as quais agregam valor à pessoa e à organização. Uma nova competência revela, inexoravelmente, que a pessoa aprendeu algo novo, porque mudou sua forma de atuar no trabalho (Brandão, 2008).

Visto que o desenvolvimento de competências ocorre por meio da aprendizagem, criar novas formas de prover oportunidades de aprendizagem constitui um desafio para as empresas (Casey, 1999), sobretudo porque a complexidade do ambiente organizacional provoca diferenças entre o que as pessoas sabem e o que elas precisam aprender (Pozo, 2002).

As organizações, portanto, estão premidas a oferecer a seus empregados oportunidades formais de aprendizagem que, embora possam ser estruturadas em diferentes formatos e envolver ampla variedade de atividades (Sonnentag et al., 2004), são geralmente denominadas ações de treinamento, desenvolvimento e educação TD\&E (Vargas \& Abbad, 2006).

Não obstante a importância das atividades de TD\&E para promover o desenvolvimento de competências profissionais, alguns autores destacam que talvez o principal espaço de aprendizagem dentro das organizações seja o próprio ambiente de trabalho. Le Boterf (1999), por exemplo, comenta que qualquer situação de trabalho pode tornar-se uma oportunidade de aprendizagem à medida que constitui um objeto de análise, um momento de reflexão, ação e profissionalização. Talvez por isso alguns autores (Holman et al., 2001; Illeris, 2004) estejam se dedicando ao estudo de estratégias de aprendizagem informal no trabalho.

\section{Estratégias de Aprendizagem no Trabalho}

Embora a aprendizagem nas organizações seja geralmente associada a processos de TD\&E, existem outros mecanismos adotados para promovê-la (Freitas \& Brandão, 2006; Le Boterf, 1999). É comum as pessoas aprenderem em seu local de trabalho, por meio de orientações recebidas de superiores, de observações que fazem acerca do comportamento dos outros e da reflexão sobre as conseqüências do seu próprio comportamento (Pantoja \& Borges-Andrade, 2009). Parecem oportunas, então, as proposições e investigações empíricas sobre as estratégias adotadas pelas pessoas para aprender informalmente em seu trabalho.

Estratégias de aprendizagem no trabalho podem ser entendidas como práticas que as pessoas utilizam para auxiliar a aquisição de conhecimentos e habilidades em seu contexto profissional (Holman et al., 2001). Constituem esforços ativos do indivíduo para aprender algo no seu local de trabalho (Sonnentag et al., 2004). Segundo Kardash e Amlund (1991), estratégias de aprendizagem compreendem atividades de processamento cognitivo, que são adotadas pelas pessoas para adquirir, armazenar, recuperar e aplicar conhecimentos em seu contexto profissional. Weinstein e Mayer (1986), por sua vez, as definem como comportamentos e pensamentos nos quais a pessoa se engaja para promover a aprendizagem.

Apesar da existência de diferentes definições, de acordo com Pantoja e Borges-Andrade (2009) as estratégias de aprendizagem envolvem três aspectos: (a) compreendem atividades de processamento cognitivo facilitadoras da aquisição, retenção e recuperação de conhecimentos; (b) englobam comportamentos empreendidos pelo indivíduo para promover a aprendizagem de conhecimentos e habilidades; e (c) podem contribuir tanto para a aquisição, retenção e recuperação de conhecimentos como para sua aplicação em diferentes contextos.

Procurando categorizar diferentes estratégias utilizadas pelas pessoas para facilitar sua aprendizagem, alguns autores têm sugerido classificações específicas. Warr e Allan (1998), por exemplo, elaboraram uma taxonomia compreendendo nove estratégias de aprendizagem distribuídas em três categorias: cognitivas (reprodução, organização e elaboração); comportamentais (procura por ajuda pessoal, busca de ajuda em material escrito e aplicação prática); e de auto-regulação (controles emocional, da motivação e da compreensão).

Warr e Downing (2000), com o propósito de diferenciar empiricamente as estratégias de aprendizagem pro- 
postas por Warr e Allan (1998), procuraram revelar a existência de correlações entre as diversas estratégias e, paralelamente, examinar o relacionamento delas com o sucesso da aprendizagem. Construíram um instrumento composto por 45 itens, abordando as nove dimensões sugeridas por Warr e Allan (1998). O questionário foi aplicado em duas amostras, sendo uma de estudantes universitários e outra de participantes de um treinamento profissionalizante. As análises revelaram a existência de uma estrutura com oito fatores, tendo as estratégias de organização e elaboração se juntado em um único fator, denominado reflexão ativa. Os resultados revelaram que algumas estratégias, como controle emocional e controle da motivação, estavam negativamente associadas a ganhos de aprendizagem, sugerindo que a utilização delas não resulta necessariamente em melhor aprendizagem $\mathrm{e}$ que seu uso pode não ser útil para todas as pessoas (Warr \& Downing, 2000). Em escalas construídas posteriormente e validadas em contextos de trabalho (Holman et al., 2001; Pantoja, 2004), não foram incluídas variáveis relacionadas a estratégias de auto-regulação - propostas originalmente por Warr e Allan (1998) -, em geral porque estas não apresentam resultados consistentes na literatura sobre o tema (Zerbini, Carvalho, \& Abbad, 2005), que confirmem sua associação com o êxito da aprendizagem.

É o caso da medida desenvolvida por Holman et al. (2001), que validaram uma escala de estratégias de aprendizagem no call center de um banco britânico. Encontraram as categorias relacionadas a seguir, sendo que as três primeiras constituem estratégias de aprendizagem cognitivas, enquanto as três últimas referem-se a estratégias comportamentais: (a) Reprodução - repetição mental da informação, sem reflexão sobre seu significado; (b) Reflexão intrínseca - elaboração e organização de estruturas mentais que relacionam partes componentes de um trabalho; (c) Reflexão extrínseca - elaboração e organização de estruturas mentais que relacionam o trabalho a outros aspectos da organização; (d) Procura de ajuda interpessoal - busca ativa pelo auxílio de outras pessoas; (e) Procura de ajuda em material escrito - pesquisa e localização de informações em documentos, manuais, livros e outras fontes não-sociais; e (f) Aplicação prática - experimentação, tentativa de colocar em prática os próprios conhecimentos enquanto aprende.

Pantoja (2004), a partir das categorias reveladas por Holman et al. (2001), procurou validar no Brasil uma escala de estratégias de aprendizagem no trabalho, utilizando uma amostra de profissionais de diferentes ocupações. Em seu estudo, essa autora encontrou cinco categorias distintas de estratégias, tendo os itens relativos às estratégias aplicação prática e reflexão intrínseca se aglutinado em um único fator. Os demais fatores mantiveram semelhança com aqueles originalmente encontrados por Holman et al. (2001). A escala elaborada por Pantoja (2004) apresenta bons indicadores psicométricos (alfa igual ou superior a 0,80 nos cinco fatores e a maioria dos itens com cargas fatoriais superiores a 0,50 ), trazendo relevante contribuição para aprofundar estudos sobre o tema no Brasil. Essa medida, no entanto, não permitiu discriminar empiricamente as estratégias de aplicação prática (comportamental) e reflexão intrínseca (cognitiva), duas dimensões teoricamente preconizadas como distintas na literatura sobre o tema (Holman et al., 2001; Warr \& Allan, 1998; Warr \& Downing, 2000). Além disso, alguns itens das escalas desenvolvidas por Holman et al. (2001) e Pantoja (2004) parecem carecer de aprimoramento, a fim de representarem melhor a idéia de esforço empreendido pelo indivíduo para aprender algo em seu trabalho. É o caso de itens como, por exemplo, "faço meu trabalho sem pensar muito sobre ele" e "executo minhas atividades sem saber para que são necessárias" - propostos por esses autores (Holman et al., 2001, p. 677; Pantoja, 2004, p. 123) para representar a estratégia de reprodução -, cujos conteúdos não parecem descrever esforços ativos do indivíduo para aprender algo, mas sim indicar certa alienação do empregado ou deficiência em seu desempenho no trabalho.

Em decorrência, o presente estudo objetivou desenvolver e validar uma nova escala para medir o uso de estratégias de aprendizagem no trabalho, bem como verificar a freqüência com que funcionários de um banco público brasileiro utilizam tais estratégias.

\section{Método}

Nas próximas subseções são apresentadas as características da amostra, bem como os instrumentos e procedimentos adotados para coleta e análise de dados.

\section{Amostra}

Os dados foram coletados, em abril de 2008, junto a 926 gestores de agências de um banco público brasileiro, atuante em todas as regiões do Brasil e no exterior. Os participantes eram, em sua maioria, do sexo masculino (69\%), exerciam cargos de gerência média $(70 \%)$, tinham idade superior a 35 anos (72\%) e mais de 10 anos de trabalho na empresa (64\%). Possuíam curso de graduação (84\%) e pós-graduação lato ou stricto sensu (50\%) e estavam localizados em diferentes unidades da federação em todas as regiões do Brasil.

\section{Instrumentos}

Utilizou-se um questionário estruturado para a coleta de dados. A primeira parte do instrumento possuía uma apresentação, com informações sobre a pesquisa e o caráter confidencial das respostas, agradecimentos à colaboração do respondente e orientações para resposta. Em seguida, foram listadas 28 estratégias de aprendizagem, em relação às quais os respondentes deveriam assinalar a freqüência com que utilizavam cada uma no trabalho, variando de 1 (nunca faço) a 10 (sempre faço). Havia 
também, no questionário, espaço destinado à coleta de dados biográficos, como gênero, tempo de serviço no banco, escolaridade e cargo exercido.

Os 28 itens do questionário descreviam práticas utilizadas para aprender informalmente no ambiente de trabalho. Foram elaborados com base em: (a) proposições da literatura sobre o tema (Holman et al., 2001; Illeris, 2004; Pantoja, 2004; Sonnentag \& Kleine, 2000; Sonnentag et al., 2004; Warr \& Allan, 1998; Warr \& Downing, 2000); (b) análise de documentos da organização estudada, cujos conteúdos versavam sobre trilhas de aprendizagem, hábitos de leitura, práticas de gestão do conhecimento e comunicação interna; e (c) análise de depoimentos de vinte funcionários da empresa que, por meio de entrevistas, descreveram livremente as práticas e instrumentos que utilizavam para aprender no trabalho.

Para formulação dos itens do questionário foram observadas as recomendações de Richardson (1999) e Viegas (1999), de tal forma que se buscou evitar a elaboração de frases longas ou com múltiplas idéias e a utilização de expressões ambíguas, extremadas, negativas ou excessivamente técnicas.

Seguindo as proposições de Pasquali (1998), o instrumento de pesquisa foi previamente submetido à análise de conteúdo e à validação semântica. $\mathrm{Na}$ análise de conteúdo, o questionário foi apreciado por cinco juizes (pesquisadores da área de comportamento organizacional), objetivando verificar a adequação e suficiência de seus itens para representar os seis tipos de estratégias de aprendizagem indicados por Holman et al. (2001). Na validação semântica, por sua vez, realizou-se a aplicação do instrumento a uma amostra de 12 funcionários do banco, buscando-se identificar eventuais falhas ou incorreções, eliminar ambigüidades e itens sugestivos, a fim de assegurar a compreensão dos itens e da escala de avaliação por parte da população a que se destinava o questionário. Em decorrência de críticas e sugestões oferecidas pelos respondentes e pelos juízes, foram realizados ajustes no enunciado e em alguns itens.

\section{Procedimentos}

Os questionários foram remetidos, pelo malote interno do banco, no mês de março de 2008, a uma amostra aleatória de 2205 gestores de agências bancárias. Ao final de abril, foram computadas 926 respostas, equivalentes a um índice de retorno de $42 \%$. Foram observados, naquilo que cabia, os princípios e normas do Código Internacional de Pesquisas Sociais, da European Society for Opinion and Marketing Research (Esomar, 2005), de forma que os dados foram coletados por meio da cooperação voluntária dos respondentes, sem qualquer desvantagem para eles, respeitando-se o seu direito de privacidade e garantindo-lhes que as informações prestadas não seriam utilizadas para outra finalidade.

Para tabulação e análise dos dados, utilizou-se o aplicativo SPSS (Statistical Package for the Social Sciences). Foram analisadas inicialmente as estatísticas descritivas univariadas dos 28 itens da escala, a fim de identificar eventuais erros de digitação. Foi identificado apenas um valor fora da amplitude normal da escala de avaliação ( 1 a 10 pontos), o qual foi corrigido pela consulta à resposta assinalada no questionário. As médias e desvios padrão revelaram-se plausíveis. Os coeficientes de variação (desvio padrão dividido pela média) mostraram-se superiores a 0,001 , sugerindo não serem necessárias transformações (Neiva, Abbad, \& Tróccoli, 2007). Verificou-se que três participantes possuíam mais de $10 \%$ de respostas ausentes, razão pela qual foram excluídos das análises posteriores. Outros doze sujeitos também foram excluídos, porque assinalaram a mesma resposta para todos os itens (respostas invariadas). Todas as variáveis, por sua vez, revelaram possuir menos de $1 \%$ de dados ausentes.

Visando identificar a existência de casos extremos multivariados, utilizou-se a distância de Mahalanobis. Apurou-se que 30 respondentes constituíam outliers multivariados, o que poderia produzir impacto sobre a matriz de correlações, por diminuir ou ampliar a magnitude das associações entre variáveis (Neiva et al., 2007). Em decorrência, as análises posteriores foram realizadas com e sem os outliers na amostra, a fim de examinar seu impacto. Embora os resultados produzidos fossem essencialmente os mesmos, em termos de estrutura fatorial, a manutenção dos outliers prejudicava as cargas fatoriais de alguns itens. Optou-se então por excluí-los, visto que a eliminação desses casos extremos não prejudicaria muito o tamanho da amostra. Com a exclusão dos outliers multivariados e dos casos com respostas invariadas ou com mais de $10 \%$ de dados ausentes, a amostra ajustada totalizou 881 sujeitos.

Depois, foram examinados os requisitos necessários para se proceder à análise fatorial. No que diz respeito ao tamanho necessário da amostra, utilizou-se o critério proposto por Pasquali (2005) e Tabachnick e Fidell (1989), ou seja, o de que deve haver pelo menos cinco respondentes para cada variável observada. Este requisito foi cumprido, visto que a amostra ajustada totalizou 881 sujeitos, isto é, mais de 31 respondentes para cada item da escala. Em relação à normalidade das distribuições, foram analisados os histogramas e os índices de skewness e kurtosis das variáveis, tendo a significância desses valores (ao nível de 0,05 ) sido verificada por meio do escore $Z$, como recomendam Hair, Anderson, Tatham e Black (2005). Tais índices revelaram a ausência de distribuição normal em boa parte das variáveis, sendo mais comum a moderada assimetria negativa. Como a análise fatorial, no entanto, é razoavelmente robusta a violações do pressuposto da normalidade (Laros, 2005), principalmente em grandes amostras - com mais de 200 sujeitos (Hair et al., 2005; Pasquali, 2006), optou-se por realizar os procedimentos subseqüentes utilizando os dados originais, sem a sua transformação.

Procurou-se, em seguida, verificar a linearidade das relações entre as variáveis, extraindo-se gráficos de dispersão bivariada entre pares de itens e examinando a 
magnitude das correlações entre eles. Essa análise, em geral, indicou a presença de associação linear entre as variáveis verificadas, duas a duas, tendo as relações entre pares apresentado intensidades bem variadas. Embora em alguns pares fosse alta a intensidade dessas correlações, nenhuma delas mostrou-se próxima ou superior a 0,90 , o que sugere ausência de multicolinearidade (Pasquali, 2005), outro pressuposto da análise fatorial.

Uma vez examinados tais aspectos, procurou-se verificar a fatorabilidade da matriz de correlações e identificar quantos fatores essa matriz comportava, o que é descrito a seguir.

\section{Resultados}

Para verificar a fatorabilidade da matriz de correlações, foram analisados diferentes aspectos. A inspeção visual da matriz revelou a existência de um bom percentual (cerca de 55\%) de correlações iguais ou superiores a 0,30 e algumas chegaram a 0,70 , índices que sugerem a fatorabilidade dos dados. Obteve-se uma medida de adequação amostral Kaiser-Meyer-Olkin (KMO) igual a 0,93, que indica uma ótima adequação dos dados à análise fatorial (Pasquali, 2005). Examinando a matriz de correlações anti-imagem, verificou-se que os quadrados das correlações múltiplas $\left(R^{2}\right)$, em sua grande maioria, eram superiores a 0,90 , o que indica suficiente relação entre as variáveis para se proceder a uma análise fatorial. Além disso, o fato de o determinante da matriz de correlações ser igual a 0,0000000881 (praticamente zero) representa outro indicativo de fatorabilidade (Pasquali, 2005).

Para determinar o número de fatores, utilizou-se a análise dos componentes principais (PC) e, como critérios, os autovalores iguais ou superiores a um (Kaiser, 1960, citado por Pasquali, 2005), o scree plot (Cattell, 1966, citado por Laros, 2005), a análise paralela (Horn, 1965, citado por Pasquali, 2005), o percentual da variância explicada pelos fatores (Harman, 1967), a variância total explicada, a matriz residual de correlações e a existência de significado teórico ou semelhança semântica entre as variáveis agrupadas em um mesmo fator.

Ponderando-se tais critérios, pôde-se verificar que a estrutura com cinco fatores era a mais adequada, bem como passível de interpretação, uma vez que as variáveis se agruparam em torno das categorias de estratégias de aprendizagem definidas por Holman et al. (2001) e Pantoja e Borges-Andrade (2009): reflexão extrínseca; reflexão intrínseca; reprodução; busca de ajuda interpessoal; busca de ajuda em material escrito; e aplicação prática. Diferença básica entre a estrutura empírica obtida e essas seis categorias foi o fato de as variáveis representativas das estratégias reflexão extrínseca e reflexão intrínseca terem se unido em um único fator, conforme exposto adiante.

Empregou-se o método de fatoração dos eixos principais (Principal Axis Factoring), com rotação oblíqua (promax), para extração dos fatores, uma vez que havia correlações entre eles. Uma variável foi eliminada porque não apresentou carga fatorial igual ou superior a 0,30 em nenhum dos cinco fatores. Os fatores extraídos explicam $62,3 \%$ da variância dos itens componentes da matriz fatorial, percentual considerado bastante razoável. Foi verificada a adequação (goodness-of-fit) da análise fatorial realizada, examinando se a matriz das correlações residuais de fato continha apenas resíduos. Verificou-se a existência de 28 resíduos (abaixo da diagonal da matriz dos resíduos - $R_{\text {res }}$ ), equivalentes a $7 \%$, com valores absolutos maiores que 0,05 , sendo a maioria destes pouco superiores a esse valor. Como é relativamente pequeno o percentual de resíduos grandes, há pouca variância comum que não é explicada pelos cinco fatores, o que sugere adequação da solução fatorial (Pasquali, 2005).

Para verificar a consistência interna dos fatores, utilizou-se o alfa de Cronbach $(\alpha)$, índice geralmente utilizado para estimação da fidedignidade das variáveis integrantes de cada fator (Pasquali, 1998). Os cinco fatores produziram alfas iguais ou superiores a 0,79 , índices que indicam ser boa a sua consistência interna (Hair et al., 2005). Nessa etapa, outra variável foi eliminada por prejudicar a consistência interna do seu fator. Como o alfa é indicativo da constância dos fatores, esses resultados sugerem ser provável que tais fatores apareçam também em outras pesquisas que se utilizem desta escala, na organização estudada.

A interpretação dos fatores, por sua vez, foi feita por meio de análise do conteúdo semântico dos itens que compõem cada fator. Pode-se observar, na Tabela 1, que as variáveis integrantes do Fator 1 dizem respeito à reflexão do indivíduo sobre partes componentes do seu trabalho, bem como à organização de estruturas mentais que relacionam o trabalho da pessoa a diferentes aspectos da organização, o que Holman et al. (2001) denominaram, respectivamente, reflexão intrínseca e reflexão extrínseca. Como tais itens agruparam-se em um único fator, este recebeu a denominação simbólica de reflexão ativa. Esse fator revelou excelente consistência interna $(\alpha=0,92)$. Seus itens apresentaram cargas fatoriais superiores a 0,40 , magnitude que, segundo Hair et al. (2005), indica boa significância estatística.

O Fator 2, por sua vez, possui cinco itens com elevadas cargas fatoriais (iguais ou superiores a 0,70 ) e ótima consistência interna $(\alpha=0,88)$, conforme Tabela 1 . Eles versam sobre a procura ativa do indivíduo pelo auxílio de outras pessoas, estratégia de aprendizagem que Holman et al. (2001) e Warr e Allan (1998) denominaram busca de ajuda interpessoal.

As variáveis integrantes do Fator 3 versam sobre a pesquisa e a localização de informações em documentos, manuais, normativos, livros e outras fontes não-sociais, o que caracteriza a estratégia de busca de ajuda em material escrito (Holman et al., 2001; Warr \& Allan, 1998). Esse fator também revelou boa consistência interna $(\alpha=$ $0,79)$ e seus cinco itens possuem elevadas cargas fatoriais (iguais ou superiores a 0,50 ), como disposto na Tabela 1 . 
Brandão, H. P. \& Borges-Andrade, J. E. (2011). Desenvolvimento e Validação de uma Escala de Estratégias de Aprendizagem no Trabalho.

Tabela 1

Estrutura Fatorial da Escala de Estratégias de Aprendizagem no Trabalho

\begin{tabular}{|c|c|c|c|c|c|c|}
\hline Variáveis & $\mathrm{F} 1$ & $\mathrm{~F} 2$ & F3 & $\mathrm{F} 4$ & F5 & $h^{2}$ \\
\hline $\begin{array}{l}20 \text { - Busco entender como diferentes aspectos do meu trabalho } \\
\text { estão relacionados entre si }\end{array}$ & 0,89 & & & & & 0,66 \\
\hline $\begin{array}{l}24 \text { - Procuro compreender como o meu trabalho está relacionado } \\
\text { aos resultados obtidos nas diferentes áreas da organização }\end{array}$ & 0,85 & & & & & 0,64 \\
\hline $\begin{array}{l}28 \text { - Tento conhecer como as diferentes áreas da organização } \\
\text { estão relacionadas entre si }\end{array}$ & 0,81 & & & & & 0,67 \\
\hline $\begin{array}{l}27 \text { - Busco compreender as relações entre as demandas feitas por } \\
\text { outras áreas da organização e a finalidade do meu trabalho }\end{array}$ & 0,77 & & & & & 0,69 \\
\hline $\begin{array}{l}11 \text { - Tento compreender como a atuação das diferentes áreas } \\
\text { da organização influencia a execução do meu trabalho }\end{array}$ & 0,76 & & & & & 0,57 \\
\hline $\begin{array}{l}23 \text { - Para melhor execução do meu trabalho, reflito sobre como ele } \\
\text { contribui para atender as expectativas dos clientes }\end{array}$ & 0,70 & & & & & 0,57 \\
\hline $\begin{array}{l}10 \text { - Quando faço meu trabalho, penso em como ele está relacionado } \\
\text { ao negócio e às estratégias da organização }\end{array}$ & 0,66 & & & & & 0,50 \\
\hline $\begin{array}{l}19 \text { - Para aprimorar a execução de minhas atividades, procuro } \\
\text { compreender melhor cada procedimento e tarefa do meu trabalho }\end{array}$ & 0,52 & & & & & 0,61 \\
\hline $\begin{array}{l}07 \text { - Analisando criticamente a execução do meu trabalho, tento } \\
\text { compreendê-lo melhor }\end{array}$ & 0,41 & & & & & 0,47 \\
\hline $\begin{array}{l}22 \text { - Consulto colegas de trabalho mais experientes, quando tenho } \\
\text { dúvidas sobre algum assunto relacionado ao meu trabalho }\end{array}$ & & 0,81 & & & & 0,60 \\
\hline $\begin{array}{l}01 \text { - Busco ajuda dos meus colegas quando necessito de informações } \\
\text { mais detalhadas sobre o trabalho }\end{array}$ & & 0,80 & & & & 0,57 \\
\hline $\begin{array}{l}14 \text { - Peço ajuda aos meus colegas de equipe quando necessito } \\
\text { aprender algo sobre meu trabalho }\end{array}$ & & 0,79 & & & & 0,60 \\
\hline $\begin{array}{l}21 \text { - Procuro obter novos conhecimentos e informações consultando } \\
\text { colegas de outras equipes }\end{array}$ & & 0,73 & & & & 0,62 \\
\hline $\begin{array}{l}02 \text { - Quando tenho dúvidas sobre algo no trabalho, consulto colegas } \\
\text { de outras áreas da empresa }\end{array}$ & & 0,70 & & & & 0,52 \\
\hline $\begin{array}{l}13 \text { - Consultando informações disponíveis na intranet, busco } \\
\text { compreender melhor as atividades que executo no trabalho }\end{array}$ & & & 0,78 & & & 0,55 \\
\hline $\begin{array}{l}17 \text { - Para obter as informações de que necessito para o trabalho, } \\
\text { leio informativos e matérias publicadas na agência de notícias }\end{array}$ & & & 0,71 & & & 0,46 \\
\hline $\begin{array}{l}12 \text { - Quando tenho dúvidas sobre algo no trabalho, procuro ajuda } \\
\text { em publicações, informativos, fascículos e relatórios da empresa }\end{array}$ & & & 0,69 & & & 0,47 \\
\hline $\begin{array}{l}18 \text { - Visando obter informações importantes à execução do meu } \\
\text { trabalho, consulto a Internet }\end{array}$ & & & 0,62 & & & 0,42 \\
\hline $\begin{array}{l}16 \text { - Quando estou em dúvida sobre algo no trabalho, consulto } \\
\text { normativos e instruções editadas pela organização }\end{array}$ & & & 0,50 & & & 0,41 \\
\hline $\begin{array}{l}08 \text { - Visando executar melhor minhas atividades de trabalho, busco } \\
\text { repetir automaticamente ações e procedimentos memorizados }\end{array}$ & & & & 0,86 & & 0,53 \\
\hline $\begin{array}{l}06 \text { - Para melhor execução do meu trabalho, procuro seguir sempre } \\
\text { os mesmos procedimentos }\end{array}$ & & & & 0,68 & & 0,39 \\
\hline $\begin{array}{l}09 \text { - Para executar melhor o meu trabalho, procuro repetir mentalmente } \\
\text { informações e conhecimentos recém-adquiridos }\end{array}$ & & & & 0,61 & & 0,42 \\
\hline $\begin{array}{l}05 \text { - Para aprimorar a execução do meu trabalho, busco memorizar } \\
\text { dados ( } \mathrm{n}^{\circ} \text { de rubricas, contas, transações em sistemas, etc) }\end{array}$ & & & & 0,60 & & 0,35 \\
\hline 03 - Experimento na prática novas formas de executar o meu trabalho & & & & & 0,66 & 0,46 \\
\hline $\begin{array}{l}25 \text { - Procuro aprimorar algum procedimento de trabalho, } \\
\text { experimentando na prática novas maneiras de executá-lo }\end{array}$ & & & & & 0,62 & 0,63 \\
\hline 26 - Testo novos conhecimentos aplicando-os na prática do meu trabalho & & & & & 0,56 & 0,63 \\
\hline Autovalor & 9,86 & 3,03 & 1,94 & 1,43 & 1,19 & \\
\hline$\%$ Variância Explicada & 35,22 & 10,81 & 6,92 & 5,11 & 4,27 & \\
\hline Número de Itens & 9 & 5 & 5 & 4 & 3 & \\
\hline Alpha de Cronbach & 0,92 & 0,88 & 0,79 & 0,79 & 0,82 & \\
\hline
\end{tabular}

Nota. Foram omitidas as cargas fatoriais inferiores a 0,40 . 
O Fator 4 é composto por itens relativos à memorização e à repetição mental de informações, sem reflexão sobre seu significado, o que caracteriza a estratégia de aprendizagem denominada reprodução (Holman et al., 2001; Pantoja \& Borges-Andrade, 2009; Warr \& Allan, 1998). Seus quatro itens possuem ótimas cargas fatoriais (iguais ou superiores a 0,60 ), conforme mostra a Tabela 1. O alfa desse fator é igual a 0,79, indicando boa consistência interna.

Por fim, o Fator 5 foi denominado aplicação prática, expressão utilizada por Holman et al. (2001) e Pantoja e Borges-Andrade (2009) para designar a tentativa do indivíduo de aprender por meio da experimentação, de colocar em prática os seus conhecimentos enquanto aprende. $\mathrm{O}$ alfa igual a 0,82 indica boa consistência interna. As três variáveis componentes desse fator também possuem boas cargas fatoriais, como pode ser visto na Tabela 1.

Verificou-se também que os fatores não eram independentes entre si, visto que, como mostra a Tabela 2 , há razoável correlação entre eles. Como os cinco fatores relacionam-se positivamente entre si, tal ocorrência insinua serem eles fatores primários, ou seja, subfatores de um fator de segunda ordem. Em decorrência, esse fator de segunda ordem foi extraído e teve a sua consistência analisada, utilizando-se para tanto as 26 variáveis (dispostas na Tabela 1) integrantes dos fatores primários. Nessa nova extração, forçada em apenas um fator, foi utilizada a mesma matriz de correlações e o método de fatoração dos eixos principais (PAF), seguindo-se as recomendações de Pasquali (2005). O fator de segunda ordem extraído apresentou itens com boas cargas fatoriais, variância total explicada de $37 \%$ e alfa de 0,92 .

Procurou-se, por fim, extrair os escores fatoriais, calculando-se a média dos itens que compõem cada fator primário, conforme sugerido por Pasquali (2005). A Tabela 3 mostra as médias e desvios padrão dos cinco fatores de primeira ordem.

Tabela 2

Matriz de Correlações entre os Cinco Fatores Primários

\begin{tabular}{lccccc}
\hline & Fator 1 & Fator 2 & Fator 3 & Fator 4 & Fator 5 \\
\hline Fator 1 & 1 & & & & \\
Fator 2 & 0,46 & 1 & & & \\
Fator 3 & 0,69 & 0,41 & 1 & & \\
Fator 4 & 0,23 & 0,38 & 0,23 & 1 & \\
Fator 5 & 0,60 & 0,44 & 0,54 & 0,25 & 1 \\
\hline
\end{tabular}

Nota. Correlações significantes ao nível de 0,05.

Tabela 3

Escores Fatoriais

\begin{tabular}{lcc}
\hline Fatores Extraídos & Média & Desvio Padrão \\
\hline Fator 1: Reflexão ativa & 8,07 & 1,09 \\
Fator 2: Busca de ajuda interpessoal & 8,23 & 1,30 \\
Fator 3: Busca de ajuda em material escrito & 8,00 & 1,30 \\
Fator 4: Reprodução & 7,02 & 1,58 \\
Fator 5: Aplicação prática & 7,80 & 1,27 \\
\hline
\end{tabular}

Os fatores 1 e 4 representam estratégias cognitivas de aprendizagem, enquanto os demais se referem a estratégias comportamentais, conforme taxonomia defendida por Holman et al. (2001) e Warr e Allan (1998). Pode-se observar, na Tabela 3, que as estratégias de aprendizagem mais utilizadas pelos respondentes são a busca de ajuda interpessoal e a reflexão ativa, enquanto a menos utilizada é a reprodução. Há relativa homogeneidade nas freqüências de uso dessas estratégias, à exceção da estratégia de reprodução, em que foi revelada maior variabilidade nas respostas $(D P=1,58)$.

\section{Discussão e Conclusões}

Pela análise realizada, a solução com a extração de cinco fatores primários revelou-se a mais adequada. Os fatores extraídos representam as estratégias de: reflexão ativa; busca de ajuda interpessoal; busca de ajuda em material escrito; reprodução; e aplicação prática.

Esse resultado, quando comparado ao obtido por Holman et al. (2001), evidencia que, na estrutura empírica aqui revelada, houve redução do número de fatores. Isso ocorreu porque, diferente do que era esperado, os itens 
relativos às estratégias de reflexão extrínseca e reflexão intrínseca uniram-se em um único fator. Embora tais estratégias sejam conceitualmente distintas, este estudo não revelou empiricamente diferenças entre elas. Como ambas constituem estratégias cognitivas de aprendizagem, os respondentes parecem ter entendido tais itens como representativos de uma mesma prática adotada por eles para auxiliar a aquisição de conhecimentos e habilidades no trabalho. Em estudo anterior (Warr \& Downing, 2000), as variáveis relativas a essas estratégias de aprendizagem também se uniram em um único fator, o qual recebeu dos autores a mesma denominação simbólica aqui adotada: reflexão ativa.

Talvez a diferença entre a estrutura aqui encontrada e aquela obtida por Holman et al. (2001) seja decorrente de particularidades do papel ocupacional exercido pelos integrantes das amostras pesquisadas. Enquanto no presente estudo os participantes exerciam papéis gerenciais de razoável complexidade, a pesquisa conduzida por Holman et al. (2001) teve como sujeitos os empregados de um call center, atividade de caráter menos complexo e mais rotineiro. Como o exercício de funções gerenciais muitas vezes exige do profissional o estabelecimento de múltiplas interrelações entre elementos do trabalho, geralmente envolvendo diferentes atores (clientes, subordinados, acionistas e parceiros, por exemplo), é provável que os participantes deste estudo tenham entendido não ser possível dissociar a reflexão extrínseca da intrínseca. Pode ser, então, que a estrutura fatorial de medidas de estratégias de aprendizagem varie em razão de características da amostra estudada, aspecto que merece ser melhor examinado em investigações futuras.

A estrutura empírica revelada por Pantoja (2004) também possuía cinco fatores, mas nela os itens relativos às estratégias de aplicação prática e de reflexão intrínseca é que se reuniram em um mesmo fator. A estrutura obtida na presente pesquisa, então, revela-se mais interessante que aquela apresentada por Pantoja (2004), visto que parece ser mais razoável a junção de variáveis relativas a duas estratégias cognitivas (reflexão intrínseca e reflexão extrínseca) que a combinação entre itens de uma estratégia cognitiva (reflexão intrínseca) e de uma comportamental (aplicação prática). Tais diferenças em estruturas fatoriais podem também ser atribuídas a particularidades de papéis ocupacionais, pois a amostra da pesquisa realizada por essa autora tinha maior variabilidade de papéis que a da presente investigação.

Quando comparados os indicadores psicométricos obtidos nesta validação com aqueles conseguidos por Pantoja (2004), não se verificam aprimoramentos substanciais: houve pequena melhoria na consistência de certos fatores (busca de ajuda interpessoal, por exemplo) e uma leve piora na de outros (reprodução e busca de ajuda em material escrito).

O principal aprimoramento realizado nesta escala diz respeito à formulação de novos itens, em especial para representar a estratégia de reprodução. Conforme men- cionado, algumas variáveis oriundas da escala de Holman et al. (2001) e utilizadas por Pantoja (2004) parecem não representar bem a idéia de aprender por meio da reprodução mental de informações. É o caso de itens como: "executo minhas atividades sem saber para que elas são necessárias"; e "faço meu trabalho sem pensar sobre ele", cujos conteúdos não parecem descrever esforços ativos do indivíduo para aprender algo, mas sim indicar certa alienação do empregado ou defíciência em seu desempenho no trabalho. Tais itens foram então eliminados da escala aqui validada e substituídos por outros como: "para executar melhor o meu trabalho, procuro repetir mentalmente informações e conhecimentos recém-adquiridos" e "para aprimorar a execução do meu trabalho, busco memorizar dados", os quais parecem representar mais adequadamente a estratégia de reprodução. Estas diferenças entre diversos itens também podem ser uma explicação para as discrepâncias encontradas entre as estruturais fatoriais obtidas por esses autores e na presente pesquisa. Um dos avanços conseguidos no presente estudo, provavelmente resultante da alteração desses itens, foi a ausência de correlações negativas entre o fator reprodução e os demais, que foram sistematicamente relatadas por outros autores.

Os cinco fatores primários extraídos revelaram-se válidos - em decorrência das boas cargas fatoriais de seus itens (todas superiores a 0,40 ) - bem como consistentes internamente, visto que seus alfas apresentaram-se iguais ou superiores a 0,79 , indicando bons índices de fidedignidade das variáveis integrantes de cada fator. Em estudos posteriores, poder-se-ia utilizar apenas os escores fatoriais para cada respondente (nos cinco fatores) e não mais os escores nos itens componentes da escala. Como tais fatores primários relacionam-se entre si, constituem subfatores de um fator de segunda ordem, que igualmente revelou boa consistência interna e itens com boas cargas fatoriais, as quais representam fontes de evidência de sua validade. O escore fatorial desse unifator também poderia ser utilizado em investigações posteriores, representando o construto estratégias de aprendizagem no trabalho, embora o uso dos cinco fatores primários permita explorar melhor os principais componentes desse construto.

Tais resultados constituem indicadores psicométricos confiáveis, de forma que o instrumento pode ser utilizado na organização estudada, com razoável segurança, tanto para conduzir investigações empíricas, quanto para orientar intervenções organizacionais, em especial para facilitar o uso de certas estratégias de aprendizagem no trabalho.

O método utilizado para realizar a presente pesquisa impõe algumas limitações a seus resultados. A utilização de amostra não-probabilística pode ter subestimado a variedade dos integrantes da população, trazendo limitações quanto à representatividade dos dados. Embora a amostra tivesse abrangência nacional - o que constitui uma vantagem -, sua circunscrição a uma organização restringe os resultados à empresa estudada, impedindo sua genera- 
lização a outros contextos organizacionais. Não há garantia absoluta de que os julgamentos feitos pelos participantes representem indicadores confiáveis do uso de estratégias de aprendizagem no trabalho, pois estimativas baseadas em auto-relato podem conter imprecisões, erros de halo e outros vieses. Acredita-se que essas limitações potenciais, embora possam restringir os resultados, não invalidam a presente investigação, até porque esta possui caráter exploratório.

Alguns estudos (Brandão \& Borges-Andrade, 2008; Sonnentag \& Kleine, 2000; Warr \& Bunce, 1995, por exemplo) mostram que a freqüência com que são utilizadas certas estratégias de aprendizagem está associada ao desenvolvimento de competências, à sua aplicação no trabalho e à melhoria do desempenho, o que reforça a importância de se realizar mais estudos sobre o tema. Considerando a relativa escassez de investigações empíricas sobre estratégias de aprendizagem no trabalho, em especial no Brasil, espera-se que a validação desta escala possa ensejar a realização de novos estudos. Utilizando esta escala, organizações e pesquisadores poderiam se dedicar a, por exemplo: (a) Verificar se a estrutura fatorial aqui revelada mantém-se em estudos conduzidos em outras organizações, não só da indústria bancária, mas também de outros segmentos de negócios, bem como em outros papéis ocupacionais; (b) Identificar que características pessoais (como idade, gênero, profissão, experiência e motivação para aprender, por exemplo) influenciam a utilização de estratégias de aprendizagem e seus resultados, em termos de desenvolvimento de competências; (c) Examinar em que medida características da equipe de trabalho (como o clima organizacional e o suporte gerencial, por exemplo) e da natureza deste trabalho (como autonomia, significado, liberdade, importância atribuída, processos e tecnologias utilizados) influenciam a utilização de estratégias de aprendizagem no trabalho e seus resultados, em termos de desenvolvimento de competências; (d) Verificar em que medida o uso de estratégias de aprendizagem no trabalho influencia os desempenhos de indivíduos, equipes e unidades organizacionais; e (e) Identificar que estratégias de aprendizagem no trabalho são mais eficazes para promover o desenvolvimento de competências de indivíduos, equipes e empresas.

Tratando-se de estudo sobre um tema emergente, espera-se que este artigo tenha oferecido contribuições teóricas e metodológicas ao estudo das estratégias utilizadas pelos indivíduos para aprender informalmente em seu ambiente de trabalho. Que ele forneça uma ferramenta de diagnóstico útil, indique caminhos e enseje a realização de outras pesquisas.

\section{Referências}

Abbad, G., \& Borges-Andrade, J. E. (2004). Aprendizagem humana em organizações de trabalho. In J. C. Zanelli, J. E. Borges-Andrade, \& A. V. Bastos (Eds.), Psicologia, organizações e trabalho no Brasil (pp. 237-275). Porto Alegre, RS: Artmed.
Brandão, H. P. (2008). Aprendizagem e competências nas organizações: Uma revisão crítica de pesquisas empíricas. Revista Eletrônica de Gestão Organizacional, 6(3), 321-342.

Brandão, H. P., \& Borges-Andrade, J. E. (2007). Causas e efeitos da expressão de competências no trabalho: Para entender melhor a noção de competência. Revista de Administração Mackenzie, 8(3), 32-49.

Brandão, H. P., \& Borges-Andrade, J. E. (2008). Learning strategies at work, perceived organizational support and professional experience as predictors of the expression of management competencies. Proceedings of the Institute of Work Psychology International Conference, UK, 1, 42-43.

Casey, C. (1999). The changing contexts of work. In D. Boud \& J. Garrick (Eds.), Understanding learning at work (pp. 15-28). London: Routledge.

European Society for Opinion and Marketing Research. (2005). International code of social research practice. Amsterdam: Author.

Freitas, I. A., \& Brandão, H. P. (2006). Trilhas de aprendizagem como estratégia de TD\&E. In J. E. Borges-Andrade, G. Abbad, \& L. Mourão (Eds.), Treinamento, desenvolvimento e educação em organizações e trabalho: Fundamentos para a gestão de pessoas (pp. 97-113). Porto Alegre, RS: Artmed.

Gagné, R. M., \& Medsker, K. L. (1996). The conditions of learning: Training applications. Belmont, CA: Thompson Learning.

Hair, J. F., Jr., Anderson, R. E., Tatham, R. L., \& Black, W. C. (2005). Análise multivariada de dados. Porto Alegre, RS: Bookman.

Harman, H. H. (1967). Modern factor analysis. Chicago: University of Chicago Press.

Holman, D., Epitropaki, O., \& Fernie, S. (2001). Understanding learning strategies in the workplace: A factor analytic investigation. Journal of Occupational and Organizational Psychology, 74(5), 675-682.

Illeris, K. (2004). A model for learning in working life. The Journal of Workplace Learning, 16(8), 431-441.

Kardash, C. M., \& Amlund, J. T. (1991). Self-reported learning strategies and learning from expository text. Contemporary Educational Psychology, 16, 117-138.

Laros, J. A (2005). O uso da análise fatorial: Algumas diretrizes para pesquisadores. In L. Pasquali (Ed.), Análise fatorial para pesquisadores (pp. 163-184). Brasília, DF: Editora da Universidade de Brasília.

Le Boterf, G. (1999). Competénce et navigation professionnelle. Paris: Éd. d'Organisation.

Matthews, J. H., \& Candy, P. C. (1999). New dimensions in the dynamics of learning and knowledge. In D. Boud \& J. Garrick (Eds.), Understanding learning at work (pp. 47-64). London: Routledge.

Neiva, E. R., Abbad, G., \& Tróccoli, B. T. (2007). Roteiro para análise fatorial de dados. Manuscrito não-publicado, Instituto de Psicologia, Universidade de Brasília, DF.

Pantoja, M. J. (2004). Estratégias de aprendizagem no trabalho e percepções de suporte à aprendizagem contínua: Uma análise multinível. Tese de Doutorado não-publicada, Instituto de Psicologia, Universidade de Brasília, DF.

Pantoja, M. J., \& Borges-Andrade, J. E. (2009). Estratégias de aprendizagem no trabalho em diferentes ocupações profissionais. Revista de Administração Contemporânea - RAC Eletrônica, 3(1), 41-62.

Pasquali, L. (1998). Psicometria: Teoria e aplicações. Brasília, DF: Editora da Universidade de Brasília. 

Trabalho.

Pasquali, L. (2005). Análise fatorial para pesquisadores. Brasília, DF: Editora da Universidade de Brasília.

Pasquali, L. (2006). Delineamento de pesquisa em ciência: Fundamentos estatísticos da pesquisa cientifica. Brasília, DF: Editora da Universidade de Brasília.

Pozo, J. (2002). Aprendizes e mestres: Nova cultura da aprendizagem. Porto Alegre, RS: Artmed.

Richardson, R. (1999). Pesquisa social: Métodos e técnicas. São Paulo, SP: Atlas.

Sonnentag, S., \& Kleine, B. M. (2000). Deliberate practice at work: A study with insurance agents. Journal of Occupational and Organizational Psychology, 73, 87-102.

Sonnentag, S., Niessen, C., \& Ohly, S. (2004). Learning at work: Training and development. International Review of Industrial and Organizational Psychology, 19, 249-289.

Tabachnick, B., \& Fidell, L. (1989). Using multivariate statistics. New York: Harper Collins.

Vargas, M. R., \& Abbad, G. (2006). Bases conceituais em treinamento, desenvolvimento e educação - TD\&E. In J. Borges-Andrade, G. Abbad, \& L. Mourão (Eds.), Treinamento, desenvolvimento e educação em organizações e trabalho: Fundamentos para a gestão de pessoas (pp. 137-158). Porto Alegre, RS: Artmed.

Viegas, W. (1999). Fundamentos de metodologia científica. Brasília, DF: Paralelo 15.

Warr, P., \& Allan, C. (1998). Learning strategies and occupational training. Internacional Review of Industrial and Organizational Psychology, 13, 83-121.

Warr, P., \& Bunce, D. (1995). Trainee characteristics and the outcomes of open learning. Personnel Psychology, 48(2), 347-375.

Warr, P., \& Downing, J. (2000). Learning strategies, learning anxiety and knowledge acquisition. British Journal of Psychology, 91(3), 311-333.

Weinstein, C. E., \& Mayer, R. E. (1986). The teaching of learning strategies. In M. C. Wittock (Ed.), Handbook of research on teaching (pp. 315-327). New York: Macmillan.

Zerbini, T., Carvalho, R. S., \& Abbad, G. S. (2005). Treinamento a distância via Internet: Construção e validação de escala de estratégias de aprendizagem. In Associação Nacional dos Programas de Pós-graduação em Administração (Ed.), Anais do XXIX Encontro Anual da Associação Nacional de Pós-graduação e Pesquisa em Administração (pp. 1-15). Brasília, DF: Associação Nacional dos Programas de Pósgraduação em Administração. 